\title{
PRÁCTICAS PRE PROFESIONALES EN CIENCIAS ADMINISTRATIVAS E INFORMÁTICAS DE LA URACCAN-NUEVA GUINEA, 2009
}

\author{
Maritza Haydeé Martínez Martínez ${ }^{[1]}$ \\ Yamilex del Socorro Aguilar Pérez ${ }^{[2]}$ \\ Mibsam Aragón Gutiérrez ${ }^{[3]}$
}

\section{Resumen}

El objeto del estudio fue la evaluación de las prácticas pre profesionales, desde la perspectiva del estudiantado, representantes de instituciones y autoridades universitarias. El enfoque fue cualitativo y por su profundidad, descriptiva, al valorar la opinión de quienes informaron en relación a la vida académica y las prácticas pre profesionales. Es de tipo transversal, semestre del 2009. La información se obtuvo a través de revisión documental, entrevistas y grupos focales.

Entre los hallazgos se destacaron aspectos favorables y desfavorables de las prácticas pre profesionales, tales como: el afianzamiento de conocimientos, oportunidad laboral y visión de la realidad laboral, establecimiento de relaciones interpersonales, falta de confianza en sí mismos e iniciativa propia, visualización de éstas como un requisito a cumplir, poca apertura y confianza de las instituciones que inciden de forma negativa en el desarrollo y alcance pleno de los objetivos en este proceso.

La carencia de normativas que regulen el proceso, la planificación, acompañamiento, evaluación y retroalimentación son vacíos sustanciales que afectan la finalidad de éstas. La diversidad de formas en que se han realizado las prácticas, vuelve más complejo el proceso por limitaciones económicas y humanas del recinto.

En base a los resultados se sugirió la elaboración de una normativa que guíe el proceso de las prácticas pre profesionales, acompañado de la asignación de recursos humanos y económicos.

Palabras claves: Prácticas pre profesionales, destreza, habilidades, evaluación.

\footnotetext{
[1] Máster en Docencia Universitaria, Asist. Área Académica. marihay17@yahoo.com

${ }^{\text {[2] }}$ Máster en Docencia Universitaria, Resp. RR HH. yamilexa_perez@hotmail.com

[3] Máster en CC. SS. con mención en investigación e intervención social, tutora de la investigación y Secretaria Académica. mibsamaragon@yahoo.com
} 


\section{Summary}

The objective of the study was to evaluate the pre-professional practices, from the perspective of the students, representatives of institutions and university authorities.

The focus was qualitative and descriptive, to be able to value the opinion of those who gave information related to the academic life and the pre-professional practices. Is transversal and it covers the semester of the year 2009. The information was gathered through document review, interviews and focus groups.

Among the findings, the pros and cons of the pre-professional practices were highlighted, such as: the strengthening of knowledge, job opportunities and vision of the working reality, establishment of relationship, lack of self-confidence and initiative, to visualize these as a requirement to comply, insufficient confidence in the institutions which impact in a negative way on the development and the achievement of the objectives in this process.

The lack of regulations to govern the process, the planning, monitoring, evaluation and feedback are substantial gaps that affect them. The different ways in which these practices have been made, makes the process more complex due to financial and human limitations in the Campus.

Based on the results, the development of rules was suggested to guide the process of the pre-professional practices, as well as the assignment of human and financial resources.

Keywords: Pre-professional practices, skills, abilities, evaluation.

\section{Introducción}

Este estudio ofrece una valoración de las prácticas pre profesionales desde tres grandes actores: los estudiantes practicantes, representantes de instituciones y autoridades universitarias, durante el segundo semestre del 2009.

Los resultados serán de utilidad para quienes han protagonizado este proceso, especialmente para la URACCAN que podrá incorporarlo en la revisión y readecuación curricular de dos de las carreras en estudio.

Las prácticas pre profesionales son una pieza clave al integrar actividades teóricas en los procesos educativos que conllevan a una práctica ocupacional, promover un ejercicio efectivo de afianzación, al considerarlo como un laboratorio próximo a la realidad laboral en la cual los estudiantes deberán ejercer sus servicios profesionales. 
Esta investigación consta de cinco capítulos. El primero trata del proceso para la realización de las prácticas pre profesionales. Un segundo muestra la relación entre las funciones que ejercen los practicantes con respecto a lo descrito en el perfil profesional de cada carrera. Un tercer capítulo contiene fortalezas y debilidades identificadas en la investigación, desde el punto de vista de quienes están involucrados con dicho proceso.

En el cuarto capítulo, se realiza una evaluación del seguimiento otorgado por la Universidad a la población estudiantil que realiza las prácticas profesionales. Y el último, contempla las sugerencias de los involucrados para mejorar las prácticas pre profesionales, tomando como referencia las debilidades encontradas en el proceso. Este capítulo contiene el principal aporte de la investigación que podrá ser retomado tanto por los estudiantes como por las autoridades universitarias para enriquecer este proceso tan importante en la formación del educando.

\section{Revisión de literatura}

Práctica pre profesional alude a la capacitación complementaria, que recibe el estudiante como parte esencial de su formación académica, las cuales deben estar vinculadas a la realidad concreta que atraviesa un país, una provincia o región determinada.

\section{La universidad pública de Lima en Perú, Universidad Nacional Agraria La Molina (UNALM; S/f.), define:}

Las prácticas pre profesionales son las actividades desarrolladas por estudiantes en el ámbito de la especialidad, con la gestión y supervisión de la facultad, a fin de consolidar la formación integral del estudiante afianzando sus destrezas y habilidades; brindándole la oportunidad para entrar en contacto formal con la realidad rural y el mercado laboral de su competencia (p. 1).

Para otros autores las prácticas representan la ventana que permite al estudiante visionar lo que posteriormente se convertirá en su realidad laboral.

\section{Para la universidad Privada Antenor Orrego:}

Las prácticas pre profesionales tienen por objetivo otorgar al estudiante la posibilidad de concretar la realidad laboral, la aplicación de los instrumentos técnico - científicos de su preparación académica, además busca ampliar los horizontes en investigación y especialización dentro del campo profesional con suficiente criterio analítico y racional (p.2).

La carrera de licenciatura en Administración de Empresas, requiere la realización de 120 horas de trabajo de terreno, para culminar estudios de pregrado (URACCAN, 2003). En el caso de Contabilidad Pública y Auditoría se exige el cumplimiento de 
120 horas de prácticas pre profesionales (URACCAN, s/f). La carrera de Informática Administrativa, requiere de 120 horas como prácticas pre- profesionales, para culminar estudios (URACCAN, 2004).

\section{Materiales y métodos}

La investigación se realizó en el municipio de Nueva Guinea, RAAS, Nicaragua, en el Recinto de la URACCAN. Es de tipo descriptivo por el nivel de profundidad con el que se describe el fenómeno, de carácter transversal por el período en estudio y con enfoque cualitativo (Barrantes, 200o).

La población se integró por el estudiantado matriculado en las carreras de Contabilidad Pública y Auditoría, Administración de Empresas e Informática Administrativa, los representantes de instituciones donde se realizan las prácticas pre profesionales y las autoridades universitarias. De esto se tomó a 4 estudiantes por carrera, 6 representantes de instituciones (3 públicas y 3 privadas), el coordinador del área en estudio y la vicerrectora. Los criterios de selección: estudiantes que hubieran culminado sus prácticas pre profesionales en el II semestre del 2009 en el área de Ciencias Administrativas, que estuvieran incluidos en el plan de seguimiento de la Universidad, representantes y/o responsables de áreas de instituciones gubernamentales y no gubernamentales donde los estudiantes hayan realizado prácticas pre profesionales, y que estuvieran incluidos en el plan de seguimiento a las prácticas, coordinador de área, vicerrectora de la universidad, por ser las instancias responsables de gerenciar todo el proceso de prácticas.

La información fue obtenida por medio de la revisión documental, entrevistas y grupos focales. Se digitó toda la información y se organizó mediante una matriz que agrupara los resultados por categorías para la realización del análisis, triangulación e interpretación de la información, utilizando el programa Word.

\section{Resultados y discusión}

\section{Prácticas pre profesionales vistas desde el practicante}

1. Se identifica la empresa.

2. Solicitud escrita o verbal a la organización.

3. Solicitud por escrito a la coordinación de área.

4. La coordinación solicita por escrito a la organización de la autorización de las prácticas pre profesionales.

5. Organización extiende constancia de culminación de prácticas. 
Según los informantes este es el proceso más frecuente para hacer las prácticas. Sin embargo, se obtuvieron dos explicaciones:

1. Las prácticas se dieron bajo una particularidad diferente que consistió en que la Alcaldía solicitó a la universidad le apoyara con estudiantes en la elaboración de unos 730 perfiles de proyectos, y una vez que se concluyera con este trabajo, se les convalidaría.

Las di en la alcaldía municipal a través de la elaboración de perfiles de proyectos, porque vinieron a la universidad a negociar para que los estudiantes que quisieran ayudarles a formular proyectos lo hiciéramos y luego la URACCAN nos iba a convalidar las prácticas pre profesionales (Grupo focal estudiantes).

Un aspecto relevante manifestado por el practicante, sobre la ventaja de hacerlas bajo esta modalidad, fue porque lo podían realizar en sus tiempos libres y en los lugares donde habitan (colonias y comarcas), siempre que pudieran conseguir una computadora para trabajar en sus casas y de no ser así, se les facilitaba acudir a un centro de Internet. Este proceso tuvo una duración de dos a tres semanas, en dependencia de la capacidad y habilidad de cada estudiante en la conclusión del perfil del proyecto.

En este caso, se puede denotar que el practicante no se detuvo a pensar en el peso académico que tienen las prácticas pre profesionales al desarrollarlas específicamente en la disciplina de su formación, debido a que optó por una metodología más fácil y rápida para cumplir con éstas, visualizándolas como un requisito a cumplir y no como un elemento esencial en su formación académica.

2. El otro caso identificado es el expuesto por una informante que manifestó haberlas realizado en su mismo centro de trabajo, por no haber conseguido autorización para ausentarse de sus labores por el período que éstas implican.

Las di donde trabajo, autorizadas por el coordinador de área y mi superior, para darlas en el área donde llevamos el control de la contabilidad: elaboración de planillas, elaboración de cheques y todo lo relativo a la contabilidad. (Grupo focal estudiantes).

La alternativa tomada por la informante, es válida cuando hay dificultades para lograr un permiso en el puesto de trabajo, en el cual deben buscarse opciones que permitan hacer las prácticas sin tener que abandonar su trabajo.

En este caso lo que no queda realmente claro, es ¿de qué manera la universidad da seguimiento a situaciones particulares como las antes mencionadas? Por otra parte, quedan ciertos vacíos respecto a ¿cómo interviene en el aprendizaje del practicante por el hecho de ser parte de la institución donde se hicieron? ¿Hasta dónde eso les 
favorece o desfavorece por el nivel de confianza que existe entre el practicante y la Institución? de acuerdo a lo planteado por la UNALM (S/f).

\section{Prácticas pre profesionales vistas desde los representantes de instituciones}

1. Solicitud por escrito al coordinador de área.

2. La solicitud es enviada a la organización.

3. La organización envía carta de aceptación o rechazo.

4. Se explican las normas y procedimientos de la institución.

5. Organización extiende constancia de culminación de prácticas.

\section{Prácticas pre profesionales vistas desde el coordinador de área}

En ocasiones gestiona con instituciones la realización de las prácticas.

1. El estudiante busca la organización para ejercer las prácticas.

2. Se permite realizarlas en la misma universidad.

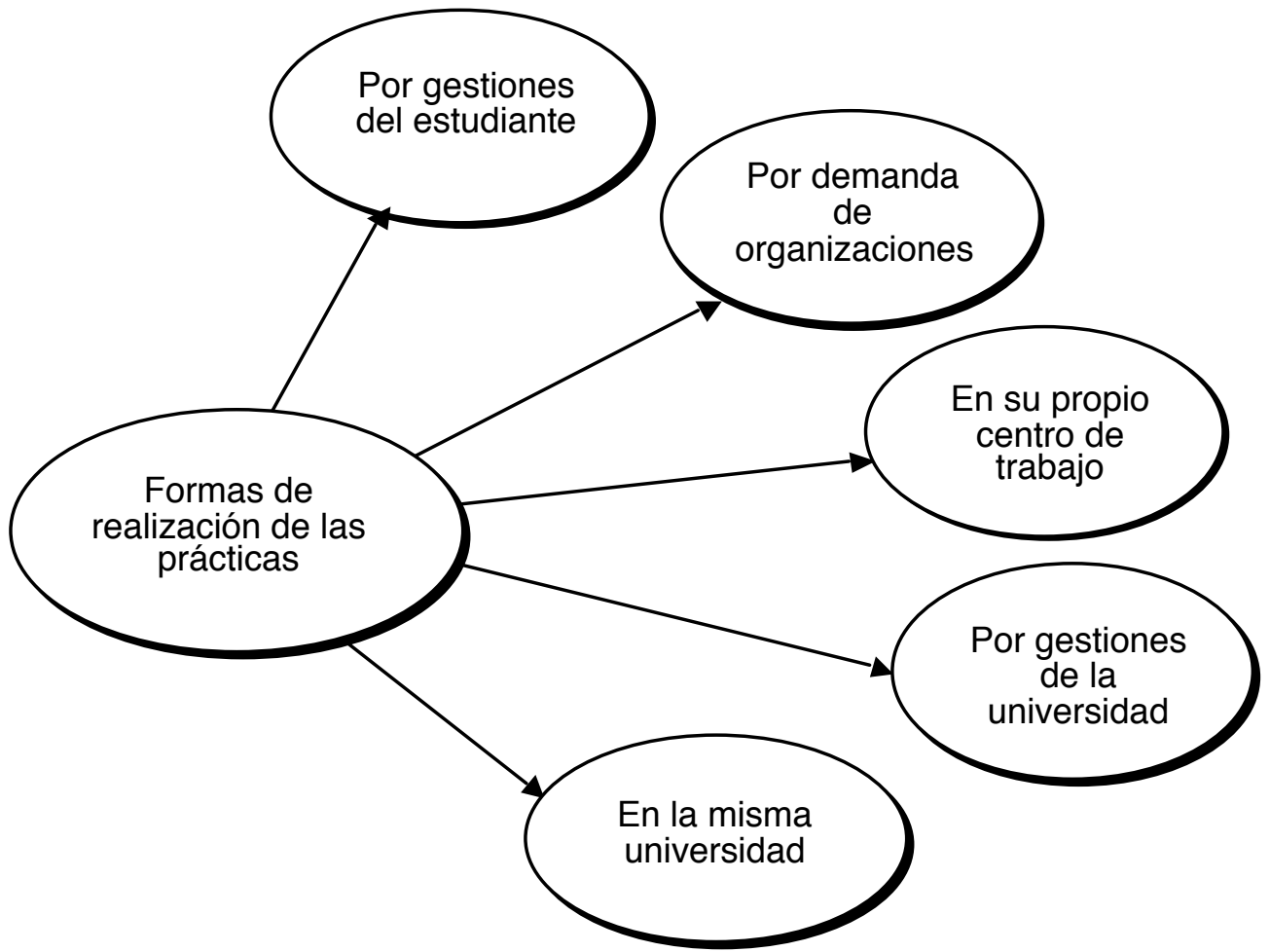

Gráfico No. 1: Formas de realizar las prácticas pre profesionales

Fuente: Elaboración propia en base a resultados de informantes. Julio 2010 


\section{Relación de funciones realizadas en el perfil profesional de la carrera en Contabilidad Pública y Auditoría}

Funciones relacionadas: Hacer registros contables en libro diario y mayor, revisar los estados financieros entre otras.

Funciones no relacionadas: actividades de logística. Existen divergencias de opiniones entre practicantes y los representantes de las instituciones. Los primeros opinan que en su mayoría realizaron actividades poco relacionadas con su profesión; sin embargo, los representantes de las instituciones, listan actividades muy vinculadas a esta profesión. Estas funciones se vuelven contradictorias con lo señalado en el perfil de la carrera, según lo establecido por URACCAN (s.f).

Administración de Empresas: La mayoría de la comunidad estudiantil opinó que las prácticas no tienen relación con su perfil profesional:

Solo han venido estudiantes de Administración y Contabilidad, en ambos casos se les enseña a manejar algunos módulos del sistema contable, adjuntar documentos soportes que generan los comprobantes del sistema, informes contables, elaboran declaración anual del Impuestos sobre la Renta (IR), informes de retenciones anuales y archivar documentos contables (Entrevista a empleadores).

Informática Administrativa: Funciones relacionadas: Organizar códigos, mantenimiento a computadoras, alimentación de sistemas.

Funciones no relacionadas: Apoyo logístico "Las funciones citadas por ambas fuentes, denotan cierta relación con el perfil profesional de la carrera, esto no contribuye a lograr el objetivo planteado por la URACCAN (2004). 
Fortalezas y debilidades de las prácticas pre profesionales

Principales fortalezas según los practicantes

\begin{tabular}{|c|c|c|}
\hline Practicantes & Representantes de instituciones & $\begin{array}{c}\text { Autoridades } \\
\text { universitarias }\end{array}$ \\
\hline $\begin{array}{l}\text { - } \text { Base teórica para desa- } \\
\text { - Utillar las prácticas. } \\
\text { - } \text { sistemación y manejo de } \\
\text { - Apoyo de la universidad. } \\
\text { - Fortalecimiento de cono- } \\
\text { cimientos. } \\
\text { - Haber culminado con las } \\
\text { - } \text { Rrácticas. } \\
\text { - Obelación teoría-práctica. } \\
\text { Periencia. }\end{array}$ & $\begin{array}{l}\text { - } \text { Descongestión y disminución } \\
\text { de la carga laboral institucional, } \\
\text { lo que difiere de lo expuesto por } \\
\text { la universidad privada: Antenor } \\
\text { Orrego (2008). } \\
\text { - Disposición de los y las estudian- } \\
\text { tes por aprender. } \\
\text { - Confianza y acompañamiento } \\
\text { constante al practicante. } \\
\text { - Algunas instituciones les brinda } \\
\text { la información requerida para su } \\
\text { - } \text { Proceso de prácticas } \\
\text { - Credibilidad y prestigio de la } \\
\text { universidad. }\end{array}$ & - Fuente de Empleo \\
\hline
\end{tabular}

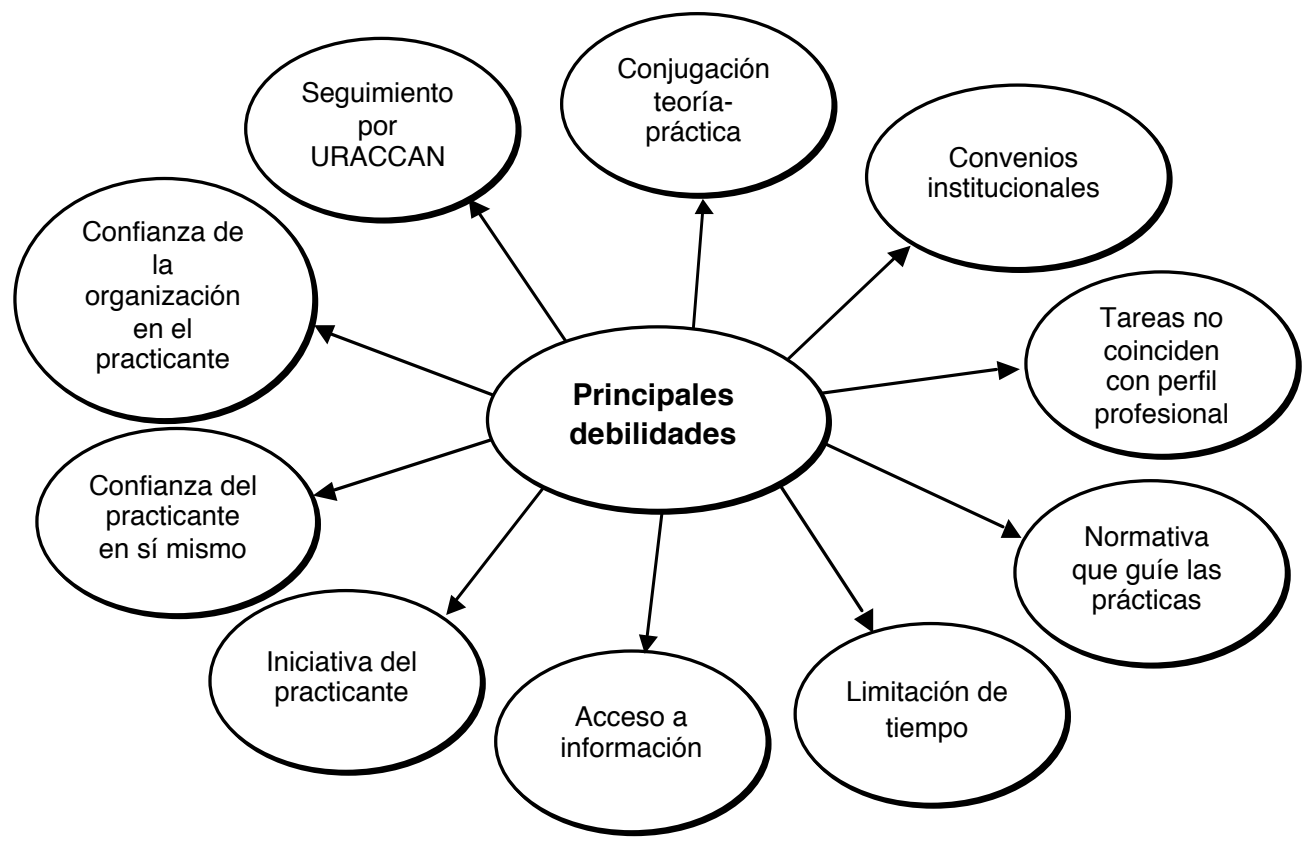

Gráfico No.2: Principales debilidades en las prácticas pre profesionales Fuente: Elaboración propia, en base a resultados de los informantes, Julio 2010 


\section{Evaluación de las prácticas pre profesionales}

Practicantes, representantes de instituciones y autoridades universitarias coinciden en que se carece de retroalimentación de este proceso y que solamente se cuenta con formatos que son facilitados a las organizaciones para su debido llenado, conforme su criterio, pero no se hace una reflexión entre los actores involucrados, que conlleve a las mejoras de los resultados.

\section{Propuestas para el desarrollo de las prácticas pre-profesionales}

- Normar el proceso de las prácticas pre profesionales.

- Seguimiento sistemático por la URACCAN.

- Establecimiento de convenios específicos para desarrollarlas.

- Previo a las prácticas pre profesionales capacitar y/u orientar al estudiante.

- Hacer diferenciación de tiempo para quienes tienen experiencias y quienes no la tienen, así lo confirma la universidad Andina (2009).

- URACCAN debe mantener una relación estrecha con las instituciones que permiten al estudiante dar sus prácticas.

- Planificación de los estudiantes que deben dar las prácticas para que las organizaciones no se saturen.

- Evaluación del proceso donde participen: URACCAN, los practicantes y la organización.

\section{Conclusiones}

Existe una valoración positiva respecto a la importancia de las prácticas por ser un espacio que permite al estudiantado ponerse en contacto con la realidad laboral y contrastar conocimientos teóricos y enfrentar la realidad con sus habilidades y destrezas. Sin embargo, este proceso ha carecido de planificación, inducción, seguimiento, evaluación y retroalimentación que contribuyan a cumplir con la finalidad de las mismas.

La diversidad de formas en que éstas se han desarrollado, deja en evidencia la flexibilidad institucional y la necesidad de normativas que guíen este proceso y conduzcan a resultados más significativos. Las limitaciones de recursos humanos y económicos de la Universidad imposibilita el acompañamiento adecuado a este proceso, así mismo la ausencia de convenios colectivos que permitan fortalecerlas.

El estudiantado considera que las prácticas no han llenado sus expectativas, porque las funciones que desempeñan no se relacionan estrechamente con su carrera y el tiempo para su desarrollo es relativamente corto. 
Existen divergencias sustanciales entre los distintos actores en la forma de visualizar las prácticas pre profesionales, porque el representante de institución las mira como una actividad que les permite descongestionar la carga laboral y ahorrar recursos, el estudiantado lo ve como un requisito a cumplir y la universidad como una actividad más que deben llevar a cabo los practicantes como parte de la culminación de estudios.

\section{Lista de referencia}

Barrantes (2000). Investigación un camino al conocimiento. 2da. ed. Costa Rica. Editorial Universidad Estatal a Distancia. p.64, 7 1-72.

URACCAN (2003). Currículum de lwa carrera de administración de Empresas. Managua-Nicaragua.

URACCAN (2004). Currículum de la carrera de Informática Administrativa (URACCAN), Bilwi, RAAN-Nicaragua.

URACCAN (s.f.). Perfil Académico URACCAN. Nueva Guinea, RAAS, Nicaragua

\section{Recursos electrónicos}

Calienni, M., A. \& Moledda, M. (2007). Las prácticas pre-profesionales colectivas en la carrera de Lic. En Servicio Social de la Universidad Nacional de Mar de Plata. Lógicas $y$ fracturas del escenario/territorio en las que se desarrollan. Recuperado el 27 de Junio de 2009. En línea: http://www.gias.com.ar/congreso_nacional_de_trabajo_social/Calienni, M. y otros.pdf.

Universidad Andina (2009). Prácticas preprofesionales. Recuperado el 26 de Junio de 2009. En línea: http://www.uandina.edu.pe/practicas.php

Universidad privada, Antenor Orrego (2008). Reglamento de prácticas preprofesionales. Recuperado el 27 de Junio de 2009. En Línea: http://www.upao.edu.pe/upload/ facultad/ar/Reglam_prac_preprof.

La Universidad Nacional Agraria La Molina (S.f). Reglamento de prácticas pre profesionales. Recuperado el o3 de Julio de 2009. En línea: http://www.lamolina.edu. pe/facultad/zootecnia/web2007/./Practicas.doc 